\title{
Terahertz Image Enhancing Based on the Physical Model and Multiscale Retinex Algorithm
}

qi mao

Dongbei University of Finance and Economics

yunlong zhu ( $\nabla$ zyl@fudan.edu.cn )

Fudan University

jingbo liu

Dongguan University of Technology

cixing Iv

Dongguan University of Technology

yao lu

Dongguan University of Technology

dongshan wei

Dongguan University of Technology

shihan yan

Chongqing Institute of Green and Intelligent Technology

\section{Research Article}

Keywords: physical model, multiscale retinex, $\mathrm{THz}$ images

Posted Date: April 9th, 2021

DOI: https://doi.org/10.21203/rs.3.rs-393245/v1

License: (c) (i) This work is licensed under a Creative Commons Attribution 4.0 International License.

Read Full License 


\title{
Terahertz image enhancing based on the physical model and multiscale retinex algorithm
}

\author{
Qi Mao ${ }^{1}$, Jingbo Liu ${ }^{2+}$, Yunlong Zhu ${ }^{3 *}$, Cixing Lv ${ }^{2}$, Yao Lu ${ }^{2}$, Dongshan Wei ${ }^{2}$, and Shihan Yan ${ }^{4}$ \\ ${ }^{1}$ School of Management Science and Engineering, Dongbei University of Finance and Economics, Dalian \\ 116025, China \\ ${ }^{2}$ School of Electrical Engineering and Intelligentization, Dongguan University of Technology, Dongguan \\ 523808, China \\ ${ }^{3}$ Academy for Engineering \& Technology, Fudan University, Shanghai 200433, China \\ ${ }^{4}$ Chongqing Engineering Research Center of High Resolution and Three-Dimensional Dynamic Imaging \\ Technology, Chongqing Institute of Green and Intelligent Technology, Chongqing 400714, China \\ *Corresponding authors: (zyl@fudan.edu.cn) \\ these authors contributed equally to this work
}

\section{Abstract}

To settle the THz image degradation problem, we propose an effective enhancement method based on the physical model and multiscale retinex (MSR) algorithm. The overall enhancing process involves two parts: reconstruction and enhancement. Firstly, the original $\mathrm{THz}$ images are reconstructed by a mathematical model, which is built and considered the $\mathrm{THz}$ absorption variate and Gaussian distribution of the beam. Then, the original images are processed by the proposed algorithm, which is combined the atmospheric scattering model and optimized MSR algorithm. The proposed algorithm not only recover the image scene radiance and remove haze, but also can make a compromise of the dynamic range of grayscale and edge enhancement of image. Results on a variety of $\mathrm{THz}$ images demonstrate our method can effectively improve the quality of $\mathrm{THz}$ images and retain sufficient image details.

\section{Introduction}

Terahertz imaging technology has been widely researched in the field of biomedical science, security control, and non-destructive detection [1]. Non-destructive detection of industrial products has great significance for improving production efficiency [2]. The optical inspection technology cannot observe the inside of the integrated circuit because the material of packaging cannot be penetrated. Ultrasonic testing is time-consuming and laborious, and the ultrasonic couplant used in the testing process can contaminate the products [3]. Although X-ray technology is widely used in the industrial field, its ionization characteristics can cause damage to the internal circuit structure [4]. However, the THz image degradation is still seriously affected by the fluctuation effects from the source, the intrinsic noise from the detector, and the limit of the $\mathrm{THz}$ system. The effective $\mathrm{THz}$ imaging technology has significant value in the applicant of non-destructive detection. 
In recent years, the image enhancing techniques based on image enhancement and physical models have a significant development. In the enhancing approaches based on physical models, there are several methods. Schildknecht et al. used the bind deconvolution and a point-spread function develop the biomedical image of a histopathological sample [5]. In order to distinguish counterfeit integrated circuit, Kiarash built a THz mathematical modeling for the point spread function [6]. Olivieri et al. improved the resolution of single-pixel imaging by combining nonlinear $\mathrm{THz}$ generation with time-resolved field measurements [7]. Kaiming He et al. used a dark channel prior removed the haze and recover a high quality image from a single input image [8]. These approaches produce impressive results. However, it cannot obtain high quality images and recover sufficient image details.

To enhance the $\mathrm{THz}$ image contrast and weaken these effects, many enhancing algorithms had been proposed in $\mathrm{THz}$ image processing. Trofimov et al. used high contrast correlation function to improve the image quality of the passive $\mathrm{THz}$ imaging devices [9]. Chen et al. proposed the combined approach of the discrete wavelet transform and retinex algorithm to enhance continuous wave terahertz ( $\mathrm{CW}-\mathrm{THz}$ ) scanning images contrast and denoising [10]. Wong et al. enhanced the $\mathrm{THz}$ image resolution beyond the diffraction limit, which through reconstructing the signal using sinc-envelope and feeding it into the process of a 2D blind deconvolution procedure [11]. Burford et al. applied the high-pass error function filters to obtain the balance of image clarity and signal distortion [12]. Zhang et al. proposed a THz amplitude polynomial principle component regression algorithm for the inspection of aramid-basalt hybrid composite laminates [13]. Muniyappan et al. enhanced images by using contrast limited adaptive histogram equalization method [14]. Zhang et al. distinguished different biological tissues through adopting a composite multiscale entropy approach of THz signal complexity analysis [15]. The single-scale retinex (SSR) algorithm based on a homomorphic filtering way was proposed by Jobson et al [16]. Rahman et al. proposed the multiscale retinex (MSR) algorithm by linearly weighting multiple fixed-scale color channels [17]. Although the methods have made the great improvements in the field of the $\mathrm{THz}$ image resolution, the enhanced images may amplifier noise details or generate distortion. It are still not sufficient for industrial detection.

Based on the above discussion, the physical model and image enhancement for image processing have their disadvantages. We propose an effective enhancement method based on the physical model and MSR algorithm. The overall enhancing process involves two parts: reconstruction and enhancement. Firstly, the original $\mathrm{THz}$ images are reconstructed by a mathematical model, which is built and considered the $\mathrm{THz}$ absorption variate and Gaussian distribution of the beam. The original images are still hazy. Secondly, the original images are processed by the enhancing algorithm, which is based on the atmospheric scattering model and optimized MSR algorithm. The atmospheric scattering model can recover the image scene radiance and remove haze. The optimized MSR algorithm balances the dynamic range of the grayscale and edge enhancement of the images. Results on a variety of $\mathrm{THz}$ images demonstrate our method can effectively improve the quality of $\mathrm{THz}$ images and retain sufficient image details.

The rest of this paper is organized as follows. In Section 2, the atmospheric scattering model and MSR algorithm are briefly reviewed. Section 3 shows details of the mathematical model and proposed algorithm. Section 4 presents the experimental and results, which include a comparison of quantitative analysis. Finial, Section 5 gives the conclusions of this work. 


\section{Theoretical background}

\section{A: Atmospheric scattering model}

In computer vision, it is widely used to describe the formation of blurred images using an optical attenuation model [18]. It is expressed as follows:

$$
\mathrm{I}(x)=\mathrm{t}(x) \mathrm{J}(x)+(1-t(x)) \mathrm{A}
$$

where $\mathrm{I}(x)$ is the observed image, $t(x)$ is the scene transmission map, $\mathrm{J}(x)$ is the scene radiance, and A is the global atmospheric light value. $t(x)$ describes the portion of light which is not scattered. The goal is to recover $\mathrm{J}(x), t(x)$, and $\mathrm{A}$ from $\mathrm{I}(x)$. The first term on the right-hand side is the direct attenuation and related to the media transmission coefficient. The second term is the airlight. The direct attenuation expresses the scene radiance and its decay in the medium, and the airlight describes scattered light leads to the shift of the scene colors. The degradation of image quality is exacerbated by the brightness of the scene being exhausted by fog and distant objects.

\section{B: MSR algorithm}

To better explain the advantages of the proposed method based on the MSR, we briefly present the details of MSR algorithm. According to retinex theory, the brightness perceived by the human eye depends on the illumination of the environment and the reflection of light from the surface of the object. The mathematical expression is

$$
\mathrm{I}(\mathrm{x}, \mathrm{y})=\mathrm{L}(\mathrm{x}, \mathrm{y}) \cdot R(\mathrm{x}, \mathrm{y})
$$

where $I(x, y)$ is the observed image, $L(x, y)$ is its illuminance function which depends on the source of illumination and resolve the dynamic range of the image, and $R(x, y)$ is its reflectance function which depend on the target object that carries image detail and is independent of lighting. The $R(x, y)$ represents the reflection component of a target object carrying image detail information. It corresponds to high frequency component, such as the texture information and structure of the image. The $\mathrm{L}(\mathrm{x}, \mathrm{y})$ is estimated by low-filtering the $\mathrm{I}(\mathrm{x}, \mathrm{y})$ using $G_{n}(x, y)$. The goal of retinex algorithm is to obtain the reflection image $R(\mathrm{x}, \mathrm{y})$ by removing $\mathrm{L}(\mathrm{x}, \mathrm{y})$ from observed image $\mathrm{I}(\mathrm{x}, \mathrm{y})$.

The SSR could not simultaneously deal with dynamic range or tonal contrast, and may even cause halo phenomenon. For compensating the deficiency of the SSR algorithm, the MSR algorithm adopts the sum of different weighted scales of SSR. The MSR algorithm is a balance between image color fidelity and dynamic range, which is expressed as follows.

$$
\begin{gathered}
\mathrm{R}_{M S R i}(\mathrm{x}, \mathrm{y})=\sum_{n=1}^{N} \omega_{n} \cdot\left(\ln I_{i}(x, y)-\ln G_{n}(x, y) * \ln I_{i}(x, y)\right) \\
G_{n}(x, y)=\frac{1}{2 \pi \sigma_{n}{ }^{2}} e^{-\frac{x^{2}+y^{2}}{2 \sigma_{n}^{2}}}
\end{gathered}
$$

where $\mathrm{R}_{M S R i}$ is the output of the $i$ th component image $\mathrm{I}(\mathrm{x}, \mathrm{y})$ converted using MSR algorithm, and $\mathrm{G}(x, y)$ is the Gaussian function of $\mathrm{N}$ different scales. Generally, $\mathrm{N}$ is the number of scales and set to 3 in 
actual applications. Typically, $\sigma<50$ is the small scale, $50 \leq \sigma<100$ is the medium scale, $\sigma \geq 100$ is the large scale, and $\iint \mathrm{G}_{\mathrm{n}}(\mathrm{x}, \mathrm{y}) d_{x} d_{y}=1$. The $\sum_{n=1}^{N} \omega_{n}=1$ and each value of $\omega_{n}$ is equal.

\section{Proposed methods}

\section{A: Reconstructed model}

The reconstructed model based on the $\mathrm{THz}$ absorption variate is built. The Gaussian beam distribution was considered including the numerical aperture of the THZ-TDS system, spot radius at the THz beam waist, wavelength, and distance of the waist from the target into the model. The detailed description of the $\mathrm{THz}$ reconstructed model, incorporating the Gaussian distribution [19] is as follows. In a typical THz imaging system, the $\mathrm{THz}$ beam travels over the $\mathrm{THz}$ broadband. Therefore, the effective frequency of the beam must be included as a variable in the reconstructed function. Thus, $\mathrm{I}(f)$ of the reconstructed model is represented as:

$$
\mathrm{I}(f)=\int \alpha_{0}(\omega) e^{-z \alpha_{0}(\omega)} M(f) d f
$$

where $M(\rho, z, f)$ is the Gaussian beam shape coefficient, and $\alpha_{0}$ is the absorption coefficient of the $\mathrm{THz}$ beam.

Duvillaret proposed a method in which the absorption coefficient and refractive index were calculated after the THz pulse passed through the sample [20].

$$
\begin{gathered}
n_{s}(\omega)=\frac{\varphi(\omega)}{\omega d}+1 \\
\alpha_{0}(\omega)=\frac{2}{d} \ln \left[\frac{\left(4 n_{S}(\omega)\right)}{A(\omega)\left(n_{S}(\omega)+1\right)^{2}}\right]
\end{gathered}
$$

where $A(\omega)$ is the ratio of the amplitude of the $\mathrm{THz}$ pulse through the sample and reference $\mathrm{THz}$ pulse; $d$ is the thickness of the sample; $\omega$ is the corresponding angular frequency; and $\varphi(\omega)$ is the difference in the phase between the reference and sample signals.

Jepsen demonstrated that the $\mathrm{THz}$ beam, including side lobes, is in the form of a Bessel beam or Airy disk in the THz-TDS systems [21]. As reported by Sagan, the profile of the beam becomes a pure Gaussian profile when the ratio of the Gaussian beam diameter to the diameter of the truncating aperture is set to 1 [22]. The beam source is a circular aperture lens-coupled antenna, whose output is approximated by the Gaussian illumination distribution. After leaving the circular aperture and cylindrical lens of the imaging system, this beam distribution still shows a Gaussian distribution. The THz beam can be approximately expressed by a Gaussian beam. The mathematical model of the Gaussian beam is expressed as [19]:

$$
\mathrm{M}(f)=\exp \left(-2 r^{2} /\left(\frac{0.565}{\sqrt{2 \ln 2}} \frac{k}{N A} \frac{c}{f} \sqrt{1+\left(\frac{2 \ln 2}{c \pi}\left(\frac{N A}{0.565 k}\right)^{2} f z\right)^{2}}\right)\right)
$$


where $k$ is factor determined by the truncation ratio and irradiance; NA is the numerical aperture of the THZ-TDS system; $f$ is the frequency of the THz beam; $z$ is the distance between the object and waist on the $z$ axis; and $c$ is the speed of light.

Finally, substituting Eq. (8) into Eq. (5) gives the THz image reconstructed function (Eq. (9)) as:

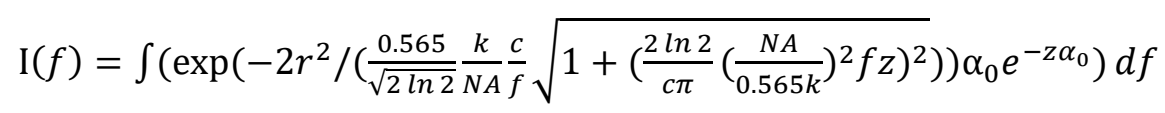

\section{B: Proposed algorithm}

The original $\mathrm{THz}$ images obtained from the reconstructed model are hazy and further enhanced. The proposed algorithm based on the atmospheric scattering model and MSR algorithm is used to enhance the $\mathrm{THz}$ images. The proposed algorithm not only recovers the image scene radiance and removes haze, but also can make a compromise of the dynamic range of grayscale and edge enhancement of the image. The algorithm scheme is described in detail.

\section{Step 1: Data input $I(\mathbf{x}, \mathbf{y})$}

The data input $l(\mathrm{x}, \mathrm{y})$ is obtained from the original THz image, which is reconstructed from the (9).

\section{Step 2: Optimized transmission $t(x)$}

$I_{\text {dark }}$ is the minimum value of three channels in each pixel. According to (9), the color of the air in a hazy image is usually very similar to the atmospheric light A. $\mu$ is set to 0.3.

$$
\begin{gathered}
\mathrm{A}=\max \left(\max \left(I_{\mathrm{dark}}(\mathrm{x}, \mathrm{y})\right)\right) \\
t(x)=1-\mu \cdot\left(I_{\mathrm{dark}}(\mathrm{x}, \mathrm{y}) / \mathrm{A}\right)
\end{gathered}
$$

Step 3: Recover the image scene radiance $I^{\prime}(\mathbf{x}, \mathbf{y})$

In order to recover the image scene radiance, the (1) is rewrote as (11). The $t_{0}$ is set to 0.1 .

$$
I^{\prime}(\mathrm{x}, \mathrm{y})=\frac{I(\mathrm{x}, \mathrm{y})-A}{\max \left(t(x), t_{0}\right)}+A
$$

\section{Step 4: Optimized MSR}

According the (4) and $\iint G_{n}(x, y) d_{x} d_{y}=1$, calculate the $G_{n}(x, y)(n=3)$ for each scale: $\sigma_{1}=$ $40, \sigma_{2}=80$, and $\sigma_{3}=150$. The $G_{n}(x, y)$ is regarded as a low-pass filter. Put the $I(x, y)$ and $\mathrm{G}_{\mathrm{n}}(\mathrm{x}, \mathrm{y})$ into (13), compute the $\mathrm{R}_{M S R}(\mathrm{x}, \mathrm{y})$. The $\alpha$ and $\beta$ are respectively added and regarded as the gain factor and adjustment coefficient in optimized MSR. In this work, the weighting coefficients $\omega_{n}$ are $0.333,0.333$, and 0.334 .

$$
R(\mathrm{x}, \mathrm{y})=\beta \cdot \ln \left(\frac{\alpha}{3}+1\right) \cdot \sum_{n=1}^{N} \omega_{n} \cdot\left(\ln I^{\prime}(x, y)-\ln G_{n}(x, y) * \ln I^{\prime}(x, y)\right)
$$


Where the $\alpha$ is $\frac{1}{6} \cdot \sqrt{M}<\alpha<\frac{1}{4} \cdot \sqrt{M}$ and $\beta$ is 0.8 . $M$ is the number of pixels of observed image.

\section{Step 5: Data output $R^{\prime}(\mathbf{x}, \mathbf{y})$}

$$
R^{\prime}(\mathrm{x}, \mathrm{y})=e^{R(\mathrm{x}, \mathrm{y})}
$$

Obtain the enhanced image from the $R^{\prime}(\mathrm{x}, \mathrm{y})$.

\section{Results}

The experiment is implemented on the T-Gauge 5000 manufactured by Advanced Photonics. The focal length of the TDS system is $75 \mathrm{~mm}$ and the diameter of the lens is $38 \mathrm{~mm}$. The point-by-point raster scanning is adopted in this work. The scanning step size and speed of the mobile station were $0.25 \mathrm{~mm}$ and $50 \mathrm{~mm} \mathrm{~s}-1$, respectively. The signal noise ratio of $\mathrm{THz}$ wave above $1 \mathrm{THz}$ is small, and the available frequency is range from 0.3 to $0.8 \mathrm{THz}$. The original $\mathrm{THz}$ images are performed by the different approaches, including the contrast-limited adaptive histogram equalization (CLAHE), grey level transformation (GLT), multiscale retinex (MSR), and our method. Our specimens were STC89C52RC IC, PCB board, copper-coated iron bookmarks of flower and butterfly.

Quantitative evaluation of image enhancement methods is discussed in the following. Several indicators are used to quantitatively evaluate the performance of the different image enhancement methods. For example, the standard deviation (SD) is mainly used to measure the overall contrast of the image. The entropy (EN) is mainly used to measure the expected value of a random variable in an image. In other words, EN reflects the degree of chaos in grey-scale map. The spatial frequency (SF) is an indicator of the intensity of gray mutation in an image. For the image, the edge part of the image is the abrupt part. The more rapid the mutation, the sharper the edge. The peak signal-to-noise ratio (PSNR) describes the mean square error (MSE) relationship between the original image and the enhanced image using the enhancement method. The MSE represents the expected error between the original image and enhanced image.

\section{A: IC imaging}

In Figure. 1, we show the enhanced results of $\mathrm{THz}$ IC images. The original image is relatively blurry with low image contrast. The high image contrast of the enhanced image is achieved from the proposed algorithm. The proposed algorithm obtains a better quality with high image contrast, clear IC pins, and a rational white balance in the grayscale images. In Figure. 1 (b) and (d), the image using the CLAHE and MSR methods are close and slightly worse in the sight of visual sense. The Figure. 1(c) shows good image contrast and therefore the overall enhanced image looks darker. As shown in Table 1, the EN of the image using the GLT method is larger than other methods and therefore the PSNR is large. Correspondingly, the PSNR represented is low. Comparing the original THz image and enhanced images, the image in Figure. 1 (b), (c), and (d) is overall slightly bad white balance and low contrast. 
The IC X-ray image is shown in Figure. 2 (a). The Figure. 2 (b) shows the enhanced IC image using the proposed algorithm. According to the results, the sizes of the outside pins are highly consistent with those obtained from the X-ray image. For example, the values of rulers X1 and T1 are $49.68 \mathrm{~mm}$ and $50.18 \mathrm{~mm}$, respectively, with a $1 \%$ margin of error. The values of rulers $\mathrm{X} 2$ and $\mathrm{T} 2$ are $3.92 \mathrm{~mm}$ and $4.35 \mathrm{~mm}$, respectively, with an error margin of $10 \%$. The values of rulers $X 3$ and $T 3$ are $3.3 \mathrm{~mm}$ and $3.48 \mathrm{~mm}$, respectively, with an error margin of $5 \%$. The values of rulers X4 and T4 are $13.66 \mathrm{~mm}$ and $14.9 \mathrm{~mm}$, respectively, with an error margin of $8 \%$. Given these results, it can be concluded that the proposed algorithm is better.
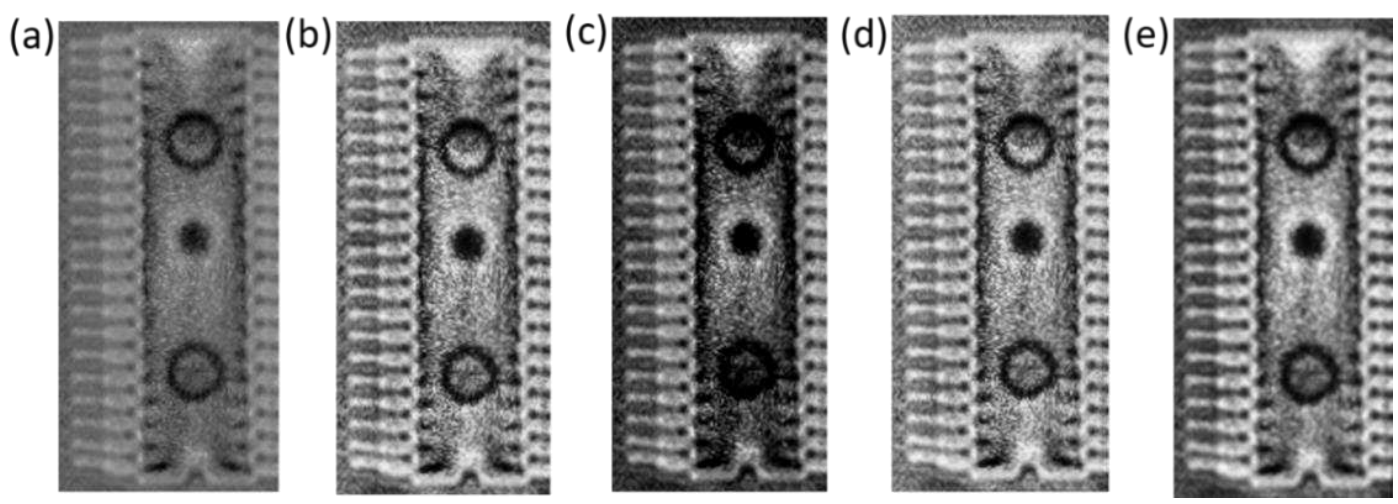

Figure. 1. (a) Original IC THz image; (b), (c), (d), (e) was the enhancement IC images using the CLAHE, GLT, MSR, and proposed algorithm, respectively.

Table 1. Objective assessments of the CLAHE, GLT, MSR, and proposed algorithm.

\begin{tabular}{ccccccc}
\hline $\begin{array}{c}\text { Imag } \\
\mathrm{e}\end{array}$ & Metric & Original & CLAHE & GLT & MSR & $\begin{array}{c}\text { proposed } \\
\text { algorithm }\end{array}$ \\
\hline \multirow{4}{*}{ IC } & SD & 38.12 & 46.99 & 54.33 & 46.18 & 53.06 \\
& EN & 6.54 & 7.35 & 7.39 & 7.35 & 7.53 \\
& SF & 27.04 & 32.72 & 31.71 & 32.29 & 35.5 \\
& MSE & - & $0.89 \times 10^{3}$ & $1.24 \times 10^{3}$ & $1.19 \times 10^{3}$ & $0.81 \times 10^{3}$ \\
& PSNR & - & 18.59 & 17.17 & 17.62 & 19.02 \\
\hline
\end{tabular}

\section{B: $P C B$ board imaging}

The enhanced THz images of the PCB board are shown in Figure. 2. The size is $8.5 \mathrm{~cm} \times 9.5 \mathrm{~cm}$, which is fabricated according to USAF1951. The original image is slightly blurry with low image contrast. However, the finer lines look blur together. The high image contrast and signal to noise ratio are achieved from the proposed algorithm. It can obtain the grayscale image, which is equipped with high image contrast, clear boundaries of the finer lines, and a rational white balance. In Figure. 2 (b) and (d), the image contrasts using the CLAHE and MSR methods are also close and comparatively worse in the sight of visual sense. The Figure. 2 (c) is better at good image contrast and lower noise. However, the overall image is 
excessively saturated and the finest lines are almost disappeared. The brightness and saturation of the Figure. 2 (e) are controlled well. As shown in Table 2, the EN of the image using the GLT method is larger than images using the CLAHE and MSR methods. The SF of the image enhanced by MSR is the highest and therefore shows a lot of noise and distortion. Correspondingly, its PSNR represented is lowest. Comparing the original $\mathrm{THz}$ image and enhanced images, the proposed algorithm shows a very well ability of balance of the image contrast, white balance, and signal to noise ratio.
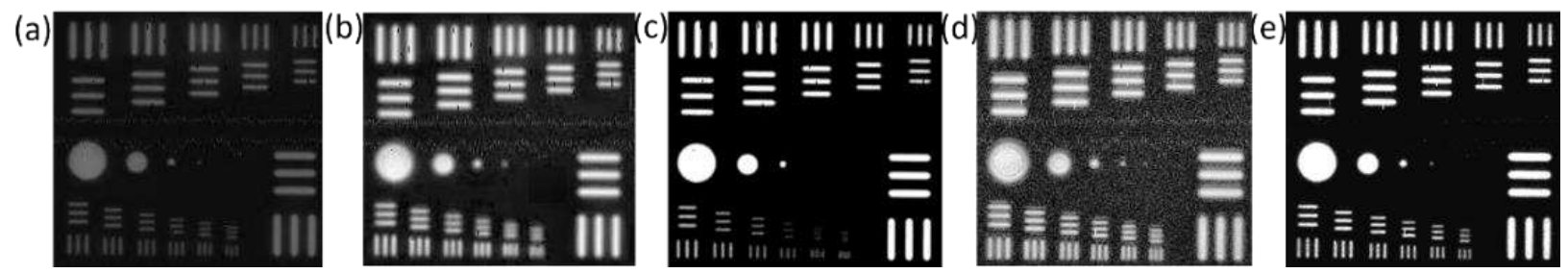

Figure. 2. (a) Original THz image; (b), (c), (d), (e) was the enhancement PCB images using the CLAHE, GLT, MSR, and proposed algorithm, respectively.

Table 2. Objective assessments of the CLAHE, GLT, MSR, and proposed algorithm.

\begin{tabular}{ccccccc}
\hline Image & Metric & Original & CLAHE & GLT & MSR & $\begin{array}{c}\text { proposed } \\
\text { algorithm }\end{array}$ \\
\hline \multirow{3}{*}{ PCB } & SD & 51.17 & 55.76 & 61.61 & 55.79 & 62.53 \\
board & EN & 5.6 & 6.34 & 1.09 & 7.3 & 1.67 \\
& SF & 22.64 & 20.26 & 26.53 & 43.47 & 29.91 \\
& MSE & - & $0.61 \times 10^{3}$ & $0.37 \times 10^{3}$ & $1.56 \times 10^{3}$ & $0.18 \times 10^{3}$ \\
& PSNR & - & 20.2 & 22.44 & 16.19 & 25.22 \\
\hline
\end{tabular}

\section{C: Flower bookmark imaging}

The enhanced results of the THz flower bookmark images are shown in Figure. 3 . The size is $7.2 \mathrm{~cm} \times 5 \mathrm{~cm}$ $\times 0.05 \mathrm{~cm}$, including the stamen and texture of the petal. The original image is seriously blurry with low image contrast. The high image contrast and signal to noise ratio are achieved from our method. It can obtain a grayscale image, which is equipped with high image contrast, clear edges of the stamen and texture of the petal, and a rational white balance. In Figure. 3 (b) and (d), the image contrast obtained from the CLAHE and MSR methods are also close and slightly worse in the sight of visual sense. Although the Figure. 3 (c) shows good image contrast and lower noise. Due to the overall image is excessively saturated, the stamen and texture of the petal are almost invisible. The brightness and saturation of the Figure. 3 (e) are controlled well. As shown in Table 3, the EN of the image obtained from the GLT method is larger than it achieved from the CLAHE and MSR methods. The SF of the image enhanced by the MSR method is the highest. Therefore, it shows a lot of noise and distortion, which seriously affects the value 
of PSNR. Comparing the original THz image and enhanced images, it can be concluded that our method works well.
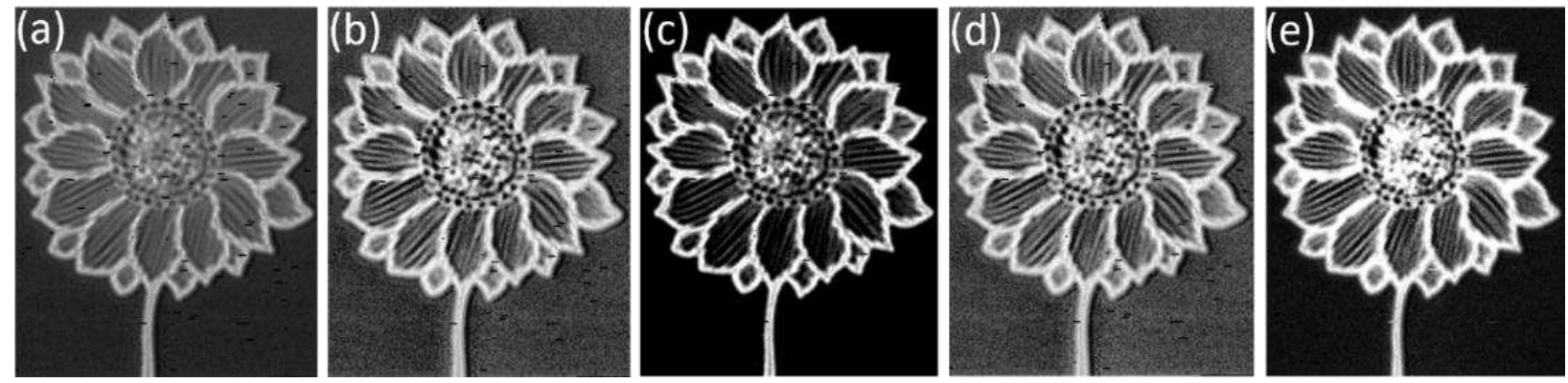

Figure. 3. (a) Original THz image; (b), (c), (d), (e) was the enhancement THz images using the CLAHE, GLT, MSR, and proposed algorithm, respectively.

Table 3. Objective assessments of the CLAHE, GLT, MSR, and proposed algorithm.

\begin{tabular}{ccccccc}
\hline Image & Metric & Original & CLAHE & GLT & MSR & $\begin{array}{c}\text { proposed } \\
\text { algorithm }\end{array}$ \\
\hline \multirow{6}{*}{ Flower } & SD & 39.83 & 50.97 & 67.97 & 49.27 & 64.96 \\
& EN & 6.59 & 7.17 & 2.6 & 7.17 & 6.8 \\
& SF & 18.53 & 36.56 & 31.48 & 36.56 & 32.54 \\
& MSE & - & $0.58 \times 10^{3}$ & $1.7 \times 10^{3}$ & $0.57 \times 10^{3}$ & $0.56 \times 10^{3}$ \\
& PSNR & - & 20.45 & 15.78 & 20.53 & 20.61 \\
\hline
\end{tabular}

\section{D: Butterfly bookmark imaging}

The enhanced THz images of the butterfly bookmark are shown in Figure. 4 . The size is $6 \mathrm{~cm} \times 6.5 \mathrm{~cm} \times$ $0.05 \mathrm{~cm}$, including the stamen and texture of the petal. The original image is seriously blurry with low image contrast and saturation. The Figure. 4 (c) shows low image contrast and saturation. In Figure. 4 (b), (c), and (d), the image contrast obtained from the CLAHE, MSR, and proposed algorithm are almost close in the sight of visual sense. As shown in Table 4, the EN of the image obtained from our proposed method is larger than others. The SF of the image obtained from the proposed algorithm is slightly smaller than it from the MSR method. But, the PSNR of the image obtained from the proposed algorithm is the best. From above discussion, the proposed algorithm shows very well able to balance the image contrast, white balance, and signal to noise ratio.
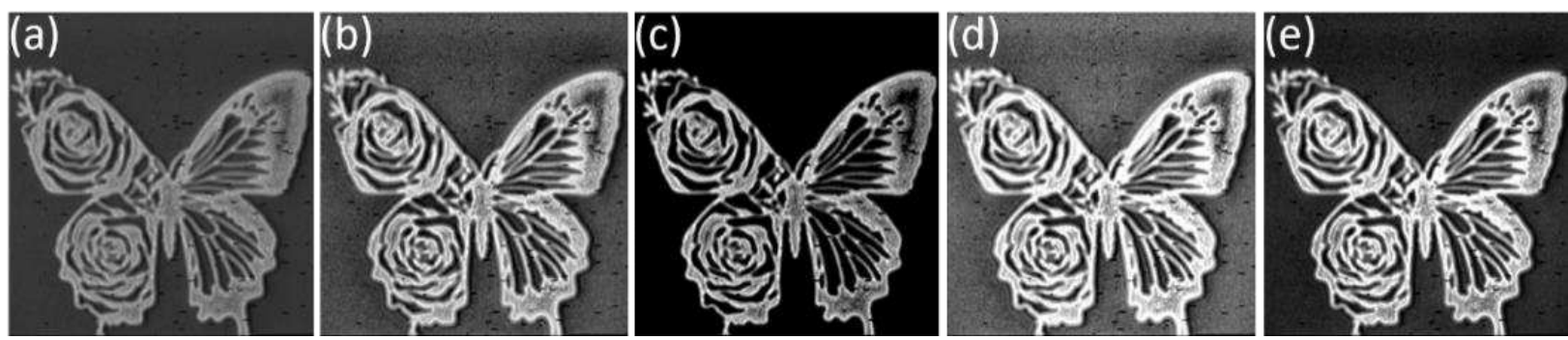

Figure 4. (a) Original image; (b), (c), (d), (e) was the enhancement images using the CLAHE, GLT, MSR, and proposed algorithm, respectively. 
Table 4. Objective assessments of the CLAHE, GLT, MSR, and proposed algorithm.

\begin{tabular}{ccccccc}
\hline Image & Metric & Original & CLAHE & GLT & MSR & $\begin{array}{c}\text { proposed } \\
\text { algorithm }\end{array}$ \\
\hline \multirow{6}{*}{ Butterfly } & SD & 36.58 & 54.87 & 67.41 & 62.56 & 72.26 \\
& EN & 5.98 & 7.26 & 2.95 & 7.19 & 7 \\
& SF & 15.88 & 29.38 & 33.58 & 34.28 & 33.44 \\
& MSE & - & 1.76 & 1.86 & 2.07 & 0.66 \\
& PSNR & - & 17.91 & 15.42 & 14.96 & 19.91 \\
\hline
\end{tabular}

\section{Discussion}

This paper presents an effective enhancement method based on the physical model and MSR algorithm. The overall enhancing process involves two parts: reconstruction and enhancement. Firstly, the original $\mathrm{THz}$ images are reconstructed by a mathematical model, which is built and considered the $\mathrm{THz}$ absorption variate and Gaussian distribution of the beam. The original THz images obtained from the reconstructed model are hazy and further enhanced. Then, the original images are processed by the proposed algorithm, which is combined the atmospheric scattering model and optimized MSR algorithm. The atmospheric scattering model can recover the image scene radiance and remove haze. The optimized MSR algorithm balances the dynamic range of the grayscale and edge enhancement of the images. Besides a subjective evaluation, the performance of the different image enhancement methods is quantitatively evaluated using several indicators, SD, EN, SF, MSE, and PSNR. The experimental results clearly show our enhancing algorithm can effectively improve the quality of $\mathrm{THz}$ images. Furthermore, this method can be applied to other fields as well, including solid-state, chemical and biological systems, and body security screening.

\section{References:}

[1] Stantchev RI, Sun B, Hornett SM, Hobson PA, Gibson GM, Padgett MJ, et al. Noninvasive, near-field terahertz imaging of hidden objects using a single-pixel detector. Sci Adv, e1600190, (2016). doi:10.1126/sciadv.1600190.

[2] Sung-Hyeon, Park, Jin-Wook, Jang, Hak-Sung, Kim. Non-destructive evaluation of the hidden voids in integrated circuit packages using terahertz time-domain spectroscopy. J Micromech Microeng, 95007, (2015)

[3] Martin E, Larato C, Clément A, Saint-Paul M. Detection of delaminations in sub-wavelength thick multi-layered packages from the local temporal coherence of ultrasonic signals. Ndt\&E Int, 280-91, (2008). doi:10.1016/j.ndteint.2007.10.013.

[4] Hertz HM, Krdel M, Dehlinger A, Seim C, Stiel H. Laboratory water-window x-ray microscopy. Optica, (2020)

[5] Schildknecht C, Kleine-Ostmann T, Knobloch P, Rehberg E, Koch M. Numerical image enhancement for THz time-domain spectroscopy., (2002) 
[6] Ahi K. Mathematical Modeling of THz Point Spread Function and Simulation of THz Imaging Systems. leee T Thz Sci Techn, 747-54, (2017). doi:10.1109/TTHZ.2017.2750690.

[7] Olivieri L, Totero Gongora JS, Pasquazi A, Peccianti M. Time-Resolved Nonlinear Ghost Imaging. Acs Photonics, 3379-88, (2018). doi:10.1021/acsphotonics.8b00653.

[8] He K, Sun J, Tang X. Single Image Haze Removal Using Dark Channel Prior. IEEE Trans Pattern Anal Mach Intell, 2341-53, (2011). doi:10.1109/TPAMI.2010.168.

[9] Schildknecht C, Kleine-Ostmann T, Knobloch P, Rehberg E, Koch M. Numerical image enhancement for THz time-domain spectroscopy., (2002)

[10] Chen L, Zhang M, Hu Q, Huang $Y$, Liang $H$, Shen C, et al. CW-THz image contrast enhancement using wavelet transform and Retinex., 9675, 96751M, (2015). doi:10.1117/12.2199505.

[11] Wong TM, Kahl M, Haring Bolívar P, Kolb A. Computational Image Enhancement for Frequency Modulated Continuous Wave (FMCW) THz Image. Journal of Infrared, Millimeter, and Terahertz Waves, 775-800, (2019). doi:10.1007/s10762-019-00609-w.

[12] Burford NM, El-Shenawee MO. Enhancement of terahertz imaging of packaged power electronic devices., 1300-01, (2015). doi:10.1109/APS.2015.7305039.

[13] Zhang H, Sfarra S, Osman A, Szielasko K, Stumm C, Sarasini F, et al. Terahertz Amplitude Polynomial Principle Component Regression for Aramid-Basalt Hybrid Composite Laminate Inspection. leee T Ind Inform, 5601-09, (2018). doi:10.1109/TII.2018.2870670.

[14] Muniyappan S, Allirani A, Saraswathi S. A novel approach for image enhancement by using contrast limited adaptive histogram equalization method., 1-06, (2013). doi:10.1109/ICCCNT.2013.6726470.

[15] Zhang R, He Y, Liu K, Zhang L, Zhang S, Pickwell-MacPherson E, et al. Composite multiscale entropy analysis of reflective terahertz signals for biological tissues. Opt Express, 23669, (2017). doi:10.1364/OE.25.023669.

[16] Jobson, Daniel, J., Rahman, Zia-ur. Properties and performance of a center/surround retinex. leee T Image Process, (1997)

[17] Rahman ZU, Jobson DJ, Woodell GW. Multiscale retinex for color rendition and dynamic range compression. Proc Spie, 183-91, (1996)

[18] Narasimhan SG, Nayar SK. Vision and the Atmosphere. Int J Comput Vision, 233-54, (2002)

[19] Ahi K, Shahbazmohamadi S, Asadizanjani N. Quality control and authentication of packaged integrated circuits using enhanced-spatial-resolution terahertz time-domain spectroscopy and imaging. Opt Laser Eng, 274-84, (2018). doi:10.1016/j.optlaseng.2017.07.007.

[20] Duvillaret L, Garet F, Coutaz JL. Highly precise determination of optical constants and sample thickness in terahertz time-domain spectroscopy. Appl Optics, 409, (1999)

[21]Jepsen PU, Keiding SR. Radiation patterns from lens-coupled terahertz antennas. Opt Lett, 807, (1995)

[22] Sagan S. Handbook of Optical and Laser Scanning, Boca Raton, USA: CRC Press; 2011. 


\section{Acknowledgements}

This work was financed by the National key research and development program of China (No. 2018YFB1702701 and No. 2018YFB1004004) and China Postdoctoral Science Foundation (No. 2018M643651).

\section{Author contributions}

Q. M carried out the laboratory work, data analysis, methodology, software, validation, data curation, and paper writing. J. L participated in conceptualization, administration, Funding acquisition. Y. Z designed the visualization. C. L and Y. L participated in supervision and project administration. D. W and S. Y carried out the investigation and resources.

\section{Competing interests}

The authors declare no competing interests. 


\section{Figures}

(a)

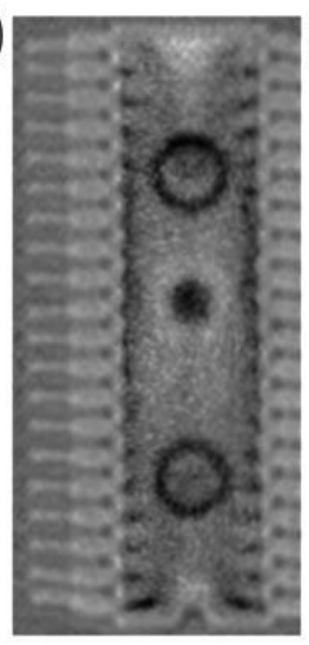

(b)

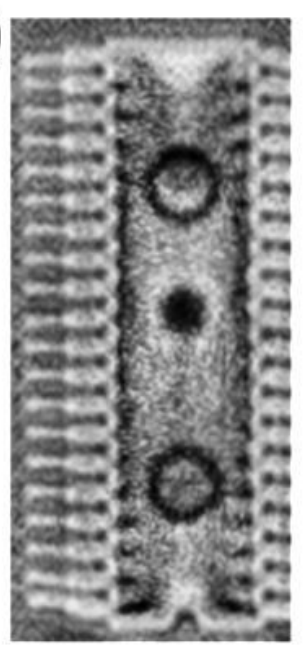

(c)

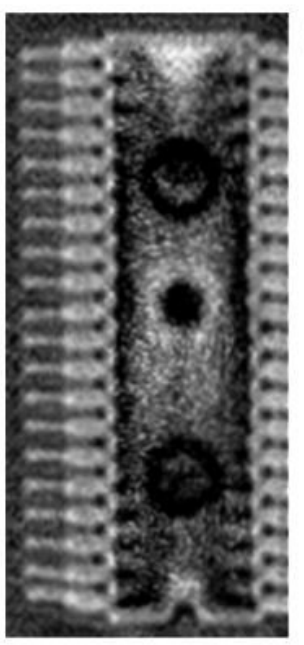

(d)

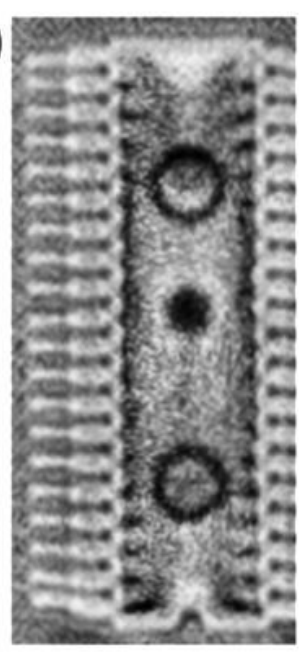

(e)

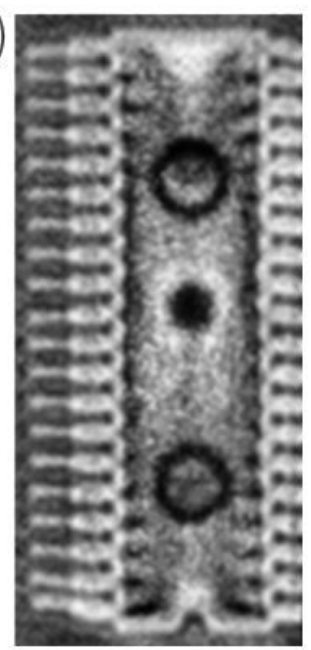

Figure 1

(a) Original IC THz image; (b), (c), (d), (e) was the enhancement IC images using the CLAHE, GLT, MSR, and proposed algorithm, respectively.

(a)
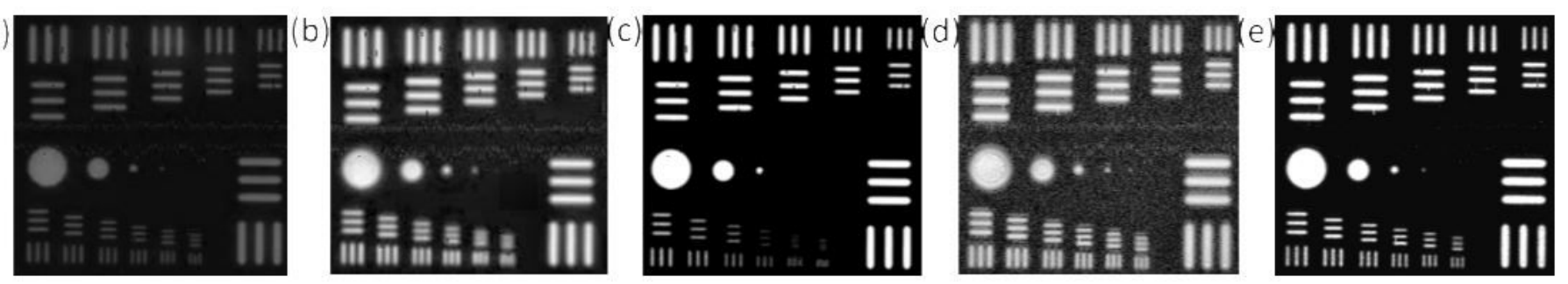

Figure 2

(a) Original THz image; (b), (c), (d), (e) was the enhancement PCB images using the CLAHE, GLT, MSR, and proposed algorithm, respectively. 

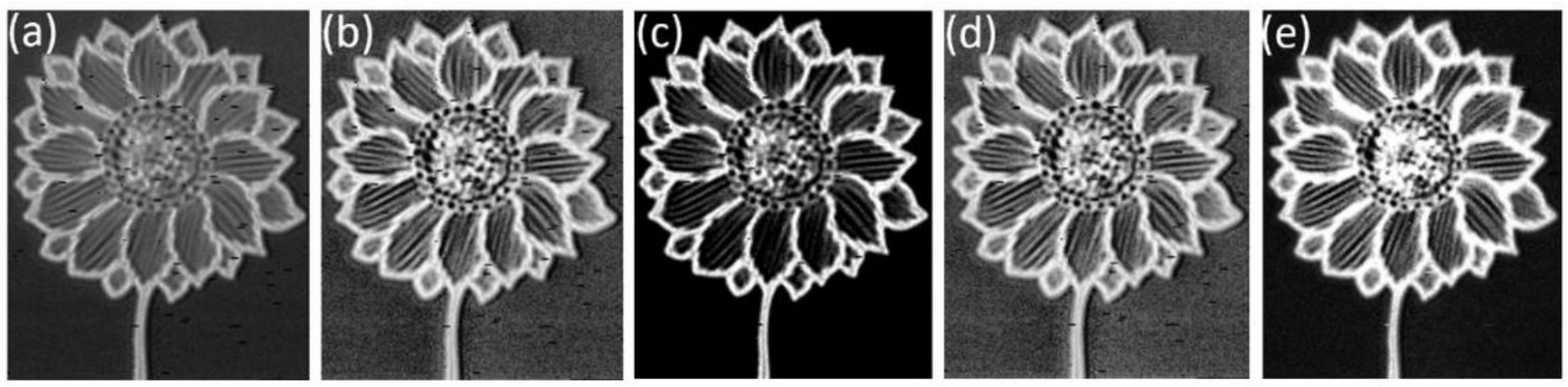

Figure 3

(a) Original THz image; (b), (c), (d), (e) was the enhancement THz images using the CLAHE, GLT, MSR, and proposed algorithm, respectively.
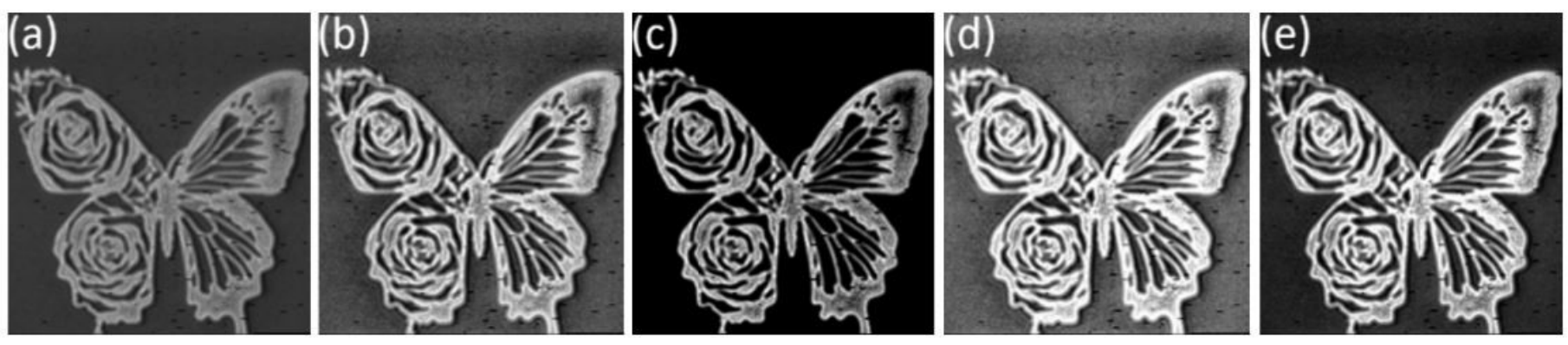

Figure 4

(a) Original image; (b), (c), (d), (e) was the enhancement images using the CLAHE, GLT, MSR, and proposed algorithm, respectively. 